\title{
UNIQUE PATH PARTITIONS: CHARACTERIZATION AND CONGRUENCES
}

\author{
CHRISTINE BESSENRODT, JØRN B. OLSSON, AND JAMES A. SELLERS
}

\begin{abstract}
We give a complete classification of the unique path partitions and study congruence properties of the function which enumerates such partitions.
\end{abstract}

\section{INTRODUCTION}

The famous Murnaghan-Nakayama formula gives a combinatorial rule for computing the value of the irreducible character of the symmetric groups $S_{n}$ labelled by the partition $\lambda$ on the conjugacy class labelled by a partition $\mu$ (see [2]). This value is the weighted sum over the $\mu$-paths in $\lambda$, as defined below, where the weight is a sign corresponding to the sum of the leg lengths of the rim hooks removed along the path.

If $\mu=\left(a_{1}, a_{2}, \ldots, a_{k}\right)$, with $a_{1} \geq a_{2} \geq \ldots \geq a_{k}>0$, and $\lambda$ are partitions of $n$, then a $\mu$-path in $\lambda$ is a sequence of partitions, $\lambda=\lambda_{0}, \lambda_{1}, \ldots, \lambda_{k}=(0)$, where for $i=1, \ldots, k$ the partition $\lambda_{i}$ is obtained by removing an $a_{i}$-hook from $\lambda_{i-1}$. As in 3, we call $\mu$ a unique path partition for $\lambda$ (or up-partition for $\lambda$ for short) if the number of $\mu$-paths in $\lambda$ is at most 1 . We call $\mu$ a up-partition if it is a $u p$-partition for all partitions of $n$.

Thus, a up-partition $\mu$ labels a conjugacy class where all non-zero irreducible character values are 1 or -1 , i.e., they are sign partitions as defined in $[3$. By [6. 7.17.4], the sign partitions $\mu$ are exactly those for which the expansion of the corresponding power sum symmetric function into Schur functions is multiplicityfree.

Note that not every sign partition is a $u p$-partition as cancellation may occur. For example, the partition $(3,2,1)$ is a sign partition, but not a up-partition, since there are two $(3,2,1)$-paths in the partition $(3,2,1)$.

In this paper, we accomplish three goals. First, we provide an explicit classification of the unique path partitions in terms of partitions we call strongly decreasing. We then discuss numerous connections between up-partitions and certain types of binary partitions. Such connections are truly beneficial; they led us to the development of a generating function for, and a recurrence satisfied by $u(n)$, the number of up-partitions of the positive integer $n$. Thanks to this link between

Date: September 3, 2012.

1991 Mathematics Subject Classification. 05A17, 11P83, $20 \mathrm{C} 30$.

Key words and phrases. binary partitions, unique path partitions, rim hooks, symmetric group, character values, congruences. 
$u p$-partitions and restricted binary partitions, we were encouraged to consider the arithmetic properties of $u(n)$. (Such a motivation is natural based on the literature that already exists on congruence properties satisfied by binary partitions. Indeed, Churchhouse [1] initiated the study of congruence properties satisfied by the unrestricted binary partition function in the late 1960's. This work was further extended by Rødseth and Sellers [4.) We close this paper by proving a number of congruence relations satisfied by $u(n)$ modulo powers of 2 .

\section{The CLASSIFICATION OF up-PARTITIONS}

We now collect the facts necessary for classifying the up-partitions in an elegant fashion. As usual, we gather equal parts together and write $i^{m}$ for $m$ parts equal to $i$ in a partition.

Lemma 2.1. (1) If $\mu=\left(a_{1}, a_{2}, \ldots, a_{k}\right)$ is a up-partition with $a_{k}=2$, then $\mu^{\prime}=$ $\left(a_{1}, a_{2}, \ldots, a_{k-1}, 1^{2}\right)$ is also a up-partition.

(2) If $\mu=\left(a_{1}, a_{2}, \ldots, a_{k}\right)$ is a up-partition with $k \geq 2$, then $\mu_{2}=\left(a_{2}, \ldots, a_{k}\right)$ is also a up-partition.

Proof. (1) follows immediately from the definition.

(2) If a partition $\lambda_{2}$ of $n-a_{1}$ has two or more $\mu_{2}$-paths then any partition of $n$ obtained by adding an $a_{1}$-hook to $\lambda_{2}$ has two or more $\mu$-paths.

Lemma 2.2. Let $\mu=\left(a_{1}, a_{2}, \ldots, a_{k}\right)$ be a partition of $n$ and $a>n$. Then $\mu$ is $a$ up-partition if and only if $\mu^{\prime}=\left(a, a_{1}, \ldots, a_{k}\right)$ is a up-partition.

Proof. By Lemma 2.1(2) we only need to show that if $\mu$ is a up-partition then also $\mu^{\prime}$ is a up-partition. Let $\lambda^{\prime}$ be a partition of $a+n$. Since $a>n, \lambda^{\prime}$ cannot contain two or more $a$-hooks. If $\lambda^{\prime}$ contains an $a$-hook, we let $\lambda$ be the partition obtained by removing it. Since by assumption $\mu$ is a $u p$-partition for $\lambda$, we get that $\mu^{\prime}$ is a $u p$-partition for $\lambda^{\prime}$.

We call an extension of a partition of $n$ by a part $a>n$ as in Lemma 2.2 strongly decreasing, or for short, an sd-extension. A partition $\mu$ obtained from a partition $\rho$ by several $s d$-extensions is then called an sd-extension of $\rho$; if $\rho=(0), \mu$ is called an $s d$-partition. As stated in [3], a partition $\mu=\left(a_{1}, a_{2}, \ldots, a_{k}\right)$ is an $s d$-partition if and only if $a_{i}>a_{i+1}+\ldots+a_{k}$ for all $i=1, \ldots, k-1$.

We have the following classification result for up-partitions:

Theorem 2.3. A partition $\mu$ is a up-partition if and only if one of the following holds:

(i) $\mu$ is an sd-partition.

(ii) $\mu$ is an sd-extension of $\left(1^{2}\right)$.

Proof. In the proof we use the well-known connection between first column hook lengths and hook removal as described in [2, Section 2.7].

As $(0)$ and $\left(1^{2}\right)$ are up-partitions, Lemma 2.2 shows that their $s d$-extensions are $u p$-partitions. Suppose that $n$ is minimal such that there exists a partition 
$\mu=\left(a_{1}, a_{2}, \ldots, a_{k}\right)$ of $n$, which is a up-partition but not an $s d$-extension of (0) or $\left(1^{2}\right)$. Obviously $k \geq 2$.

Assume $a_{2}=1$, i.e., $\mu=\left(n-k+1,1^{k-1}\right)$. If $k>3$, then $\mu$ is not a up-partition since $\left(1^{k-1}\right)$ is not. For $k=3$, only $\left(2,1^{2}\right)$ and $\left(1^{3}\right)$ are not $s d$-extensions of $\left(1^{2}\right)$, but these are not $u p$-partitions. For $k=2, \mu$ is an $s d$-partition or $\left(1^{2}\right)$.

Thus we may now assume that $a_{2}>1$. We put $\mu_{i}=\left(a_{i}, \ldots, a_{k}\right)$ and $n_{i}=\left|\mu_{i}\right|$ for $i=2, \ldots, k$. Also $n_{k+1}:=0$.

Now suppose that $a_{1}=a_{2}$. If $k=2$ then $\mu$ is not a up-partition for $\lambda=\left(a_{1}, a_{1}\right)$. If $k>2$ then $\mu$ is not a up-partition for $\lambda=\left(n-a_{1}, 1^{a_{1}}\right)$.

Thus we may now assume $a_{1}>a_{2}>1$. By Lemma $2.1 \mu_{2}=\left(a_{2}, \ldots, a_{k}\right)$ is a $u p$-partition, and thus, by minimality, it is an $s d$-extension of $(0)$ or $\left(1^{2}\right)$. Then $\mu$ cannot be an $s d$-extension of $\mu_{2}$, and hence $a_{1} \leq n_{2}$.

Now $a_{1}>a_{2}>n_{3}$ and hence $d:=a_{1}-n_{3}-1>0$. Note that $n_{2}=n_{3}+a_{2}>n_{3}+1$, and thus $\lambda=\left(n_{2}, n_{3}+1,1^{d}\right)$ is a partition of $n_{2}+n_{3}+1+d=a_{1}+n_{2}=n$. The set of first column hook lengths for $\lambda$ is $\left\{a_{1}+a_{2}, a_{1}, d, d-1, \ldots, 1\right\}$, as is easily calculated. As $d \leq n_{2}-n_{3}-1=a_{2}-1, \lambda$ has two $a_{1}$-hooks. After removing the $a_{1}$-hook in the second row we get the partition $\lambda^{\prime}=\left(n_{2}\right)$. After removing the $a_{1}$-hook in the first row we get $\left\{a_{1}, a_{2}, d, d-1, \ldots, 1\right\}$ as a set of a first column hook lengths for a partition $\lambda^{\prime \prime}$. Now $\lambda^{\prime \prime}$ has an $a_{2}$-hook in the second row. Removing it we obtain the partition $\left(n_{3}\right)$. This shows that $\mu$ is not a $u p$-partition for $\lambda$, giving a contradiction.

\section{ON up-PARTITIONS AND RESTRICTED BINARY PARTITIONS}

For each $n \in \mathbb{N}$, we denote the number of $u p$-partitions of $n$ by $u(n)$. For $t \in \mathbb{N}$, we define an $s d_{t}$-partition to be an $s d$-extension of the partition $(t)$. The following lemma is obvious.

Lemma 3.1. Let $\mu$ be a partition of $t$. There is a bijection between sd-extensions of $\mu$ and $s d_{t}$-partitions obtained by replacing all the parts of $\mu$ by one part $t$.

We denote the number of sd-partitions of $n$ by $s(n)$ and the number of $s d_{t}$ partitions of $n$ by $s_{t}(n)$ so that $s(n)=\sum_{t \geq 1} s_{t}(n)$. Combining Theorem 2.3 with Lemma 3.1 we get the following:

Corollary 3.2. For each $n \geq 1$,

$$
u(n)=s(n)+s_{2}(n) .
$$

Next, we focus our attention on $s(n)$.

Proposition 3.3. For each $n \geq 2$,

$$
s(n)=2 s_{1}(n)+s_{2}(n) .
$$

Proof. Let $\lambda=\left(a_{1}, a_{2}, \ldots, a_{k}\right)$ be an $s d_{t}$-partition, i.e., $a_{k}=t$. If we map $\lambda$ onto $\left(a_{1}, a_{2}, \ldots, a_{k}-1,1\right)$ we get a bijection between the set of all $s d_{t}$-partitions of $n$ with $t \geq 3$ and the set of all $s d_{1}$-partitions of $n$. Thus $s_{1}(n)=\sum_{t \geq 3} s_{t}(n)$. The result follows, since $s(n)=\sum_{t \geq 1} s_{t}(n)$.

Combining Corollary 3.2 and Proposition 3.3 we have the following: 
Theorem 3.4. For each $n \geq 2, u(n)$ is even. In fact,

$$
\frac{u(n)}{2}=s_{1}(n)+s_{2}(n) \text {. }
$$

Thanks to their definition, it is clear that $s d$-partitions are closely related to non-squashing partitions and binary partitions as described in [5]. A partition $\lambda=\left(a_{1}, a_{2}, \ldots, a_{k}\right)$ is called non-squashing if $a_{i} \geq a_{i+1}+\ldots+a_{k}$ for $1 \leq i \leq k-1$ and binary if all parts $a_{i}$ are powers of 2. The difference between $s d$ - and non-squashing partitions is whether or not the inequalities between $a_{i}$ and $a_{i+1}+\ldots+a_{k}$ are strict. A binary partition is called restricted (for short, an $r b$-partition) if it satisfies the following condition: Whenever $2^{i}$ is a part and $i \geq 1$ then $2^{i-1}$ is also a part. For $t \in \mathbb{N}$, an $r b_{t}$-partition is an $r b$-partition where the largest part occurs with multiplicity $t$.

With this in mind, we can naturally connect the $s d_{t}$-partitions and the $r b_{t^{-}}$ partitions.

Theorem 3.5. Let $n, t \in \mathbb{N}$. There is a bijection between the set of $s d_{t}$-partitions of $n$ and the set of $r b_{t}$-partitions of $n$.

Proof. Clearly, an $s d$-partition $\lambda=\left(a_{1}, a_{2}, \ldots, a_{k}\right)$ of $n$ is uniquely determined by the positive integers $d_{i} \in \mathbb{N}, i=1, \ldots, k$, defined by $d_{i}=a_{i}-\left(a_{i+1}+\ldots+a_{k}\right)$ for $i=1, \ldots, k-1$, and $d_{k}=a_{k}$. An easy calculation shows that with this notation $n=d_{1}+d_{2} 2+\ldots+d_{k} 2^{k-1}$. Thus if we map $\lambda$ onto the binary partition where $2^{j}$ occurs with multiplicity $d_{j+1}, j=0,1, \ldots, k-1$, we get the desired bijection.

Remark 3.6. Theorem 3.5 shows that $s(n)$ equals the number of $r b$-partitions of $n$. Let $S(q):=\sum_{n \geq 1} s(n) q^{n}$ be the generating function for $s(n)$. It is easy to write down the generating function for the number of $r b$-partitions which implies that

$$
S(q)=\sum_{i \geq 1} q^{2^{i}-1} \prod_{j=0}^{i-1} \frac{1}{1-q^{2^{j}}} .
$$

From its definition, one also gets the identity

$$
S(q)(1-q)=q\left(1+S\left(q^{2}\right)\right) .
$$

Moreover, the generating function $S_{t}(q)$ for the number of $r b_{t}$-partitions is given by

$$
S_{t}(q)=\sum_{i \geq 1} q^{2^{i}-1+(t-1) 2^{i-1}} \prod_{j=0}^{i-2} \frac{1}{1-q^{2^{j}}},
$$

and it satisfies the identity

$$
\left(S_{t}(q)-q^{t}\right)(1-q)=q S_{t}\left(q^{2}\right) .
$$

Hence, by Theorem 3.4, the generating function $U(q)$ for the number of up-partitions is then

$$
U(q)=2\left(S_{1}(q)+S_{2}(q)\right) .
$$


We now exploit this connection between $r b$-partitions and $s d$-partitions to prove a number of facts about $s(n)$ and related functions. The following results may alternatively also be proved by using the identities for the generating functions $S(q)$ and $S_{t}(q)$ stated above.

Proposition 3.7. For each $r \in \mathbb{N}$ we have

$$
\begin{aligned}
s(2 r) & =s(2 r-1) \\
s(2 r+1) & =s(2 r)+s(r) .
\end{aligned}
$$

Proof. An $r b$-partition must contain a part 1. Removing such a part from an $r b$ partition $\lambda$ of $2 r$ gives an $r b$-partition $\lambda^{\prime}$ of $2 r-1$. (A binary partition of an odd number must contain 1 as a part, so that $\lambda^{\prime}$ is still $r b$.) This map is then in fact a bijection between $r b$-partitions of $2 r$ and those of $2 r-1$.

Removing a part 1 from an $r b$-partition $\lambda$ of $2 r+1$ gives a binary partition $\lambda^{\prime}$ of $2 r$. If $\lambda^{\prime}$ has a part equal to 1 , it is an $r b$-partition and we put $\lambda^{\prime \prime}=\lambda^{\prime}$. Otherwise all parts of $\lambda^{\prime}$ are even and we may divide them all by 2 to get an $r b$-partition $\lambda^{\prime \prime}$ of $r$. The process of going from $\lambda$ to $\lambda^{\prime \prime}$ may obviously be reversed. Thus $s(2 r+1)=s(2 r)+s(r)$.

With Proposition 3.7 in mind, we define $s^{*}(r):=s(2 r)(=s(2 r-1))$ for $r \in \mathbb{N}$.

Proposition 3.8. We have $s^{*}(1)=1$ and

$$
s^{*}(r)=s^{*}(r-1)+s^{*}\left(\left\lfloor\frac{r}{2}\right\rfloor\right), \text { for } r \geq 2 .
$$

Proof. Clearly $s^{*}(1)=s(1)=1$. We prove the proposition by showing that for $r^{\prime} \in \mathbb{N}$ we have

$$
s^{*}\left(2 r^{\prime}\right)=s^{*}\left(2 r^{\prime}-1\right)+s^{*}\left(r^{\prime}\right) \text { and } s^{*}\left(2 r^{\prime}+1\right)=s^{*}\left(2 r^{\prime}\right)+s^{*}\left(r^{\prime}\right) .
$$

The equations are by definition of $s^{*}$ equivalent to

$$
s\left(4 r^{\prime}\right)=s\left(4 r^{\prime}-2\right)+s\left(2 r^{\prime}\right) \text { and } s\left(4 r^{\prime}+2\right)=s\left(4 r^{\prime}\right)+s\left(2 r^{\prime}\right) .
$$

But these are easily deduced from Proposition 3.7.

Remark 3.9. Proposition 3.8 proves that the sequence $s^{*}(n)$ is listed in [7] as A033485 and thus that the sequence $s(n)$ is listed as A040039. In particular, the comment by John McKay which appears in A40039 in [7] is confirmed.

We proceed to consider the numbers $s_{t}(r)$ of $r b_{t}$-partitions.

Proposition 3.10. Let $t \in \mathbb{N}$. We have $s_{t}(1)=s_{t}(2)=\ldots=s_{t}(t-1)=0$, $s_{t}(t)=1, s_{t}(t+1)=\ldots=s_{t}(2 t)=0$, and $s_{t}(2 t+1)=1$. Also, $s_{t}(2 r)=s_{t}(2 r-1)$ whenever $t \neq 2 r, 2 r-1$, i.e., whenever $r \neq\left\lfloor\frac{t+1}{2}\right\rfloor$.

Proof. The statements about $s_{t}(j)$ for $j \leq 2 t+1$ are trivial. The final statement is proved in analogy with Proposition 3.7. Using the notation of that proof we have the following: If we assume that $\lambda$ is $r b_{t}$ then also $\lambda^{\prime}$ is $r b_{t}$ with the exception of the case where $\lambda=\left(1^{t}\right)$. Also, if $\lambda^{\prime}$ is $r b_{t}$ then $\lambda$ is $r b_{t}$ with the exception of the case where $\lambda^{\prime}=\left(1^{t}\right)$. Thus we have $s_{t}(2 r)=s_{t}(2 r-1)$ except when $t \in\{2 r, 2 r-1\}$ 
Corollary 3.11. We have $u(1)=1, u(2)=2$, and for $r \geq 2, u(2 r)=u(2 r-1)$.

We now define

$$
s_{t}^{*}(r):=\left\{\begin{array}{cl}
s_{t}(2 r) & \text { if } r \text { is odd } \\
s_{t}(2 r-1) & \text { if } r \text { is even }
\end{array} .\right.
$$

Proposition 3.10 shows that for all $r \neq\left\lfloor\frac{t+1}{2}\right\rfloor$ we have $s_{t}^{*}(r)=s_{t}(2 r-1)=s_{t}(2 r)$. Also $s_{t}^{*}(r)=0$ for $1 \leq r \leq t, r \neq\left\lfloor\frac{t+1}{2}\right\rfloor$ and $s_{t}^{*}(t+1)=1$.

In analogy with Proposition 3.8 we have:

Proposition 3.12. For all $r \geq t+2, s_{t}^{*}(r)=s_{t}^{*}(r-1)+s_{t}^{*}\left(\left\lfloor\frac{r}{2}\right\rfloor\right)$.

Proof. The assumption on $r$ and $t$ implies that the partitions $\left(1^{r}\right)$ and $\left(1^{r-1}\right)$ are not $r b_{t}$. Therefore the bijections in the proof of Proposition 3.7 work for $r b_{t^{-}}$ partitions of $r$ as well. Thus we have the recursions

$$
\begin{aligned}
s_{t}(2 r) & =s_{t}(2 r-1) \\
s_{t}(2 r+1) & =s_{t}(2 r)+s_{t}(r) .
\end{aligned}
$$

In the case $t=1, r=3$ we have $s_{1}^{*}(3)=s_{1}(6)=1$ and $s_{1}^{*}(2)+s_{1}^{*}(1)=s_{1}(3)+s_{1}(2)=$ $1+0=1$. We assume $r \geq 4$ and write $r=4 s+i, s \in \mathbb{N}, i \in\{0,1,2,3\}$. We show

$$
\begin{aligned}
& s_{t}^{*}(4 s)=s_{t}^{*}(4 s-1)+s_{t}^{*}(2 s) \\
& s_{t}^{*}(4 s+1)=s_{t}^{*}(4 s)+s_{t}^{*}(2 s) \\
& s_{t}^{*}(4 s+2)=s_{t}^{*}(4 s+1)+s_{t}^{*}(2 s+1) \\
& s_{t}^{*}(4 s+3)=s_{t}^{*}(4 s+2)+s_{t}^{*}(2 s+1)
\end{aligned}
$$

By definition of $s_{t}^{*}$ the equations are equivalent to

$$
\begin{aligned}
& s_{t}(8 s-1)=s_{t}(8 s-2)+s_{t}(4 s-1) \\
& s_{t}(8 s+2)=s_{t}(8 s-1)+s_{t}(4 s-1) \\
& s_{t}(8 s+3)=s_{t}(8 s+2)+s_{t}(4 s+2) \\
& s_{t}(8 s+6)=s_{t}(8 s+4)+s_{t}(4 s+2) .
\end{aligned}
$$

These follow from the recursions for $s_{t}$.

Lastly, we define $w(n)=\frac{u(2 n)}{2}$. Theorem 3.4 and Proposition 3.12 yield the following:

Proposition 3.13. For each $n \geq 1, w(n)=s_{1}^{*}(n)+s_{2}^{*}(n)$. Moreover, for $n \geq 3$, $w(n)=w(n-1)+w\left(\left\lfloor\frac{n}{2}\right\rfloor\right)$, and $w(1)=w(2)=1$.

Remark 3.14. Proposition 3.13 shows that the sequence of numbers $w(n)$ is listed in [7] as A075535. The simple recurrence relation is used in the next section to prove congruence results for the numbers $w(n)$ and thus for the numbers $u(n)$ of unique path partitions.

Remark 3.15. We may consider also $w_{2}(n):=s_{3}^{*}(n)+s_{4}^{*}(n)$. Then we have $w_{2}(1)=0, w_{2}(2)=1, w_{2}(3)=0, w_{2}(4)=1$, and for $n \geq 5$

$$
w_{2}(n)=w_{2}(n-1)+w_{2}\left(\left\lfloor\frac{n}{2}\right\rfloor\right) .
$$

Similar recurrence relations are more generally valid for

$$
w_{r}(n):=s_{2 r-1}^{*}(n)+s_{2 r}^{*}(n)
$$


which starts by $w_{r}(1)=\ldots=w_{r}(r-1)=0, w_{r}(r)=1, w_{r}(r+1)=\ldots=w_{r}(2 r-1)=$ $0, w_{r}(2 r)=1$. This is an infinite family of sequences which may satisfy congruence relations similar to those satisfied by $w_{1}(n)=w(n)$.

In the next section we discuss congruences for $w(n)$ and in part also for the $w_{i}(n)$ 's.

\section{Congruences For the number of up-PARtitions}

In this section we investigate arithmetical properties of $u(n)$, the number of $u p$-partitions of $n$. Since $w(n)=\frac{u(2 n)}{2}$, any result on the $w$-sequences may be translated into a result on the $u$-sequence. In particular, as studying congruences of the $u$-sequence modulo $2 m$ is equivalent to studying the $w$-sequence modulo $m$, we will concentrate on the latter sequence.

At the start, we consider a more general situation that also covers the more general sequences defined in Remark 3.15, however, in the remaining part of this section we restrict our attention to the numbers $w(n)$.

Proposition 4.1. Let $(a(n))_{n \in \mathbb{N}}$ be a sequence with $a(c)$, $a(2 c)$ odd for some $c \in \mathbb{N}$, $a(m)$ even when $c<m<2 c$, and $a(n)=a(n-1)+a\left(\left\lfloor\frac{n}{2}\right\rfloor\right)$ for $n \geq 2 c$. Then for $n \geq c, a(n)$ is odd exactly when $n$ is of the form $2^{d} c$.

Proof. Certainly the assertion is true for $n=c$ and $n=2 c$. Assume the result holds up to some number $n=2^{r} c, r \geq 1$. Then

$$
a(n+1)=a(n)+a\left(\left\lfloor\frac{n}{2}\right\rfloor\right)=a\left(2^{r} c\right)+a\left(2^{r-1} c\right) \equiv 0 \bmod 2 .
$$

For any $k$ with $2 \leq k \leq 2^{r} c-1$, we then get by induction on $k$ that

$$
a(n+k)=a(n+k-1)+a\left(\left\lfloor\frac{n+k}{2}\right\rfloor\right) \equiv 0 \bmod 2
$$

since $2^{r-1} c<\left\lfloor\frac{n+k}{2}\right\rfloor<2^{r} c$. For $k=2^{r} c$ we then obtain

$$
a\left(2^{r+1} c\right)=a\left(2^{r+1} c-1\right)+a\left(2^{r} c\right) \equiv 1 \bmod 2 .
$$

Hence the assertion is proved.

Corollary 4.2. Let $(a(n))_{n \in \mathbb{N}}$ be as in Proposition 4.1. Let $m$ be an odd number such that $2^{b} c+1<m \leq 2^{b+1} c-1$ for some $b$. Then $a(m) \equiv a(m-2) \bmod 4$. In particular, $a(m) \equiv a\left(2^{b} c+1\right) \bmod 4$.

Proof. Since $m$ is odd, we have

$$
a(m)=a(m-1)+a\left(\left\lfloor\frac{m}{2}\right\rfloor\right)=a(m-2)+2 a\left(\left\lfloor\frac{m}{2}\right\rfloor\right) .
$$

As $m-1$ is not of the form $2^{d} c,\left\lfloor\frac{m}{2}\right\rfloor>2^{b-1} c$ is not either. Hence, $a\left(\left\lfloor\frac{m}{2}\right\rfloor\right)$ is even, and then the claim follows.

Since $w(1)=w(2)=1$, the following is immediate, and it gives corresponding congruences modulo 4 and 8 for $u(n)$ : 
Corollary 4.3. For $n \geq 1, w(n)$ is even exactly when $n$ is not a 2-power.

For any odd number $m$ such that $2^{b}+1 \leq m \leq 2^{b+1}-1$,

$$
w(m) \equiv w\left(2^{b}+1\right) \bmod 4 .
$$

Note that the first part of Corollary 4.3 implies infinitely many Ramanujan-like congruences modulo 4 satisfied by $u(n)$. To further understand the congruences of $u(n) \bmod 8$, we first focus on the 2-powers. Set $v(k)=w\left(2^{k}\right)$ for $k \in \mathbb{N}_{0}$.

Proposition 4.4. For each $k \geq 2$,

$$
v(k) \equiv 2 v(k-1)+v(k-2) \bmod 4 .
$$

Proof. Using Corollary 4.3 we have the following congruences mod 4:

$$
\begin{aligned}
v(k) & =w\left(2^{k}\right)=w\left(2^{k-1}\right)+w\left(2^{k}-1\right) \equiv w\left(2^{k-1}\right)+w\left(2^{k-1}+1\right) \\
& \equiv 2 w\left(2^{k-1}\right)+w\left(2^{k-2}\right)=2 v(k-1)+v(k-2) .
\end{aligned}
$$

Proposition 4.5. For each $k \geq 1$,

$$
v(k)=w\left(2^{k}\right) \equiv\left\{\begin{array}{ccl}
k & \bmod 8 & \text { if } k \text { is odd } \\
k+1 & \bmod 8 & \text { if } k \text { is even }
\end{array} .\right.
$$

Equivalently,

$$
v(k) \equiv 2\left\lfloor\frac{k}{2}\right\rfloor+1 \quad \bmod 8 .
$$

Proof. From the recursion formula we have

$$
\begin{aligned}
w\left(2^{k}\right) & =w\left(2^{k-1}\right)+w\left(2^{k}-1\right)=w\left(2^{k-1}\right)+w\left(2^{k-1}-1\right)+w\left(2^{k}-2\right) \\
& =w\left(2^{k-1}\right)+2 w\left(2^{k-1}-1\right)+w\left(2^{k}-3\right) \\
& \vdots \\
& =w\left(2^{k-1}\right)+2 w\left(2^{k-1}-1\right)+\ldots+2 w\left(2^{k-2}+1\right)+w\left(2^{k-1}+1\right) \\
& =2 w\left(2^{k-1}\right)+2 w\left(2^{k-1}-1\right)+\ldots+2 w\left(2^{k-2}+1\right)+w\left(2^{k-2}\right)
\end{aligned}
$$

and we now investigate sums of the form $\sum_{i=2^{d}+1}^{2^{d+1}} w(i)$, for $d \geq 1$. We want to show by induction that they are always congruent to $5 \bmod 8$; for $d=1, w(3)+w(4)=$ $2+3=5$, so the claim holds. Now we have for any $d \geq 2$ (using induction and the corollary):

$$
\begin{aligned}
\sum_{i=2^{d}+1}^{2^{d+1}} w(i) & =\sum_{i=2^{d-1}+1}^{2^{d}} w(2 i)+\sum_{i=2^{d-1}+1}^{2^{d}} w(2 i-1) \\
& =\sum_{i=2^{d-1}+1}^{2^{d}} w(i)+2 \sum_{i=2^{d-1}+1}^{2^{d}} w(2 i-1) \\
& \equiv 5+2^{d} w\left(2^{d}+1\right) \bmod 8 \\
& \equiv 5 \bmod 8 .
\end{aligned}
$$


We can now continue to compute $w\left(2^{k}\right) \bmod 8$ for $k \geq 2$ :

$$
\begin{aligned}
& w\left(2^{k}\right)=2 \sum_{i=2^{k-2}+1}^{2^{k-1}} w(i)+w\left(2^{k-2}\right) \\
& \equiv 2+w\left(2^{k-2}\right) \quad \bmod 8 \text {. }
\end{aligned}
$$

Starting with $w\left(2^{0}\right)=1=w\left(2^{1}\right)$, the assertion now follows easily.

We now obtain full information on the congruences modulo 8 for the $u$-sequence via the following result on the $w$-sequence modulo 4 .

Theorem 4.6. Let $n \in \mathbb{N}$, $n$ not a 2-power. Write $n=\sum_{i=0}^{k} 2^{n_{i}}$ with $n_{0}<n_{1}<$ $\ldots<n_{k}$. Then we have

$$
w(n) \equiv\left\{\begin{array}{llll}
0 & \bmod 4 & \text { if } n_{0} \equiv 3 \bmod 4 \\
& & \text { or } n_{0} \equiv 0 \bmod 4 \text { and } n_{k} \text { is even } \\
& \text { or } n_{0} \equiv 2 \bmod 4 \text { and } n_{k} \text { is odd } \\
2 & \bmod 4 & \text { if } n_{0} \equiv 1 \bmod 4 \\
& & \text { or } n_{0} \equiv 0 \bmod 4 \text { and } n_{k} \text { is odd } \\
& \text { or } n_{0} \equiv 2 \bmod 4 \text { and } n_{k} \text { is even }
\end{array}\right.
$$

Proof. Assume that $n_{0} \geq 1$; then $m=n-1$ is an odd number such that $2^{n_{k}}+1 \leq$ $m=n-1 \leq 2^{n_{k}+1}-1$; hence, using Corollary $4.3 w(n-1) \equiv w\left(2^{n_{k}}+1\right)=$ $w\left(2^{n_{k}}\right)+w\left(2^{n_{k}-1}\right) \bmod 4$. Then

$$
w(n)=w(n-1)+w\left(\sum_{i=0}^{k} 2^{n_{i}-1}\right) \equiv w\left(2^{n_{k}}\right)+w\left(2^{n_{k}-1}\right)+w\left(\sum_{i=0}^{k} 2^{n_{i}-1}\right) \quad \bmod 4 .
$$

If $n_{0}>1$, we can repeat the argument to obtain (using Corollary 4.3 again)

$$
\begin{aligned}
w(n) & =w(n-1)+w\left(\sum_{i=0}^{k} 2^{n_{i}-1}\right) \\
& \equiv v\left(n_{k}\right)+2 v\left(n_{k}-1\right)+v\left(n_{k}-2\right)+w\left(\sum_{i=0}^{k} 2^{n_{i}-2}\right) \bmod 4 \\
& \equiv 2 v\left(n_{k}\right)+w\left(\sum_{i=0}^{k} 2^{n_{i}-2}\right) \quad \bmod 4 \quad(\text { using Proposition 4.4) } \\
& \equiv 2+w\left(\sum_{i=0}^{k} 2^{n_{i}-2}\right) \bmod 4
\end{aligned}
$$

We now use this reduction to discuss the different cases for $n_{0}$. 
If $n_{0}=4 j-1$ for some $j \in \mathbb{N}$, then we can use the 2-step reduction above $2 j-1$ times, then the 1-step reduction, and we obtain (using Corollary 4.3 again)

$$
\begin{aligned}
w(n) & \equiv 2+w\left(2+\sum_{i=1}^{k} 2^{n_{i}-n_{0}+1}\right) \quad \bmod 4 \\
& \equiv 2+w\left(2^{n_{k}-n_{0}+1}\right)+w\left(2^{n_{k}-n_{0}}\right)+w\left(1+\sum_{i=1}^{k} 2^{n_{i}-n_{0}}\right) \\
& \equiv 2+w\left(2^{n_{k}-n_{0}+1}\right)+w\left(2^{n_{k}-n_{0}}\right)+w\left(1+2^{n_{k}-n_{0}}\right) \\
& \equiv 2+w\left(2^{n_{k}-n_{0}+1}\right)+2 w\left(2^{n_{k}-n_{0}}\right)+w\left(2^{n_{k}-n_{0}-1}\right) \\
& \left.\equiv 2+2 v\left(n_{k}-n_{0}+1\right) \equiv 0 \bmod 4 \quad \text { (using Proposition 4.4 }\right) .
\end{aligned}
$$

In the case $n_{0}=4 j+1$ for some $j \in \mathbb{N}$, we are just doing one less 2-step reduction, hence in this case it follows that $w(n) \equiv 2 \bmod 4$.

When $n_{0}=4 j$ for some $j \in \mathbb{N}$, we do again $2 j-12$-step reductions and obtain

$$
\begin{aligned}
w(n) & \equiv 2+w\left(2^{2}+\sum_{i=1}^{k} 2^{n_{i}-n_{0}+2}\right) \quad \bmod 4 \\
& \equiv 2+w\left(3+\sum_{i=1}^{k} 2^{n_{i}-n_{0}+2}\right)+w\left(2+\sum_{i=1}^{k} 2^{n_{i}-n_{0}+1}\right) \\
& \equiv 2+w\left(2^{n_{k}-n_{0}+2}+1\right)+2 \\
& \equiv w\left(2^{n_{k}-n_{0}+2}\right)+w\left(2^{n_{k}-n_{0}+1}\right) \\
& \equiv v\left(n_{k}+2\right)+v\left(n_{k}+1\right) \bmod 4 .
\end{aligned}
$$

With the previous result on the $v$-sequence, the assertion then follows.

When $n_{0}=0$, we are in the case of an odd $n$, where then (by Corollary 4.3)

$$
w(n)=w\left(1+2^{n_{k}}\right)=w\left(2^{n_{k}}\right)+w\left(2^{n_{k}-1}\right)
$$

and the result is the same as above for $n_{0}=4 j$.

When $n_{0}=4 j-2$ for some $j \in \mathbb{N}$, the result is complementary to the one above, by a shift of 2 , as stated in the assertion.

Remark 4.7. In Section 3 we have seen that the generating function $W(q)$ of $w(n)$ is the even part of $S_{1}(q)+S_{2}(q)$. The functional equations given in Remark 3.6 then yield

$$
W(q)=q+\frac{1+q}{1-q} W\left(q^{2}\right) .
$$

Iterating this equation and considering congruences modulo 2 and modulo 4 then provides a different route to the congruence results obtained above.

We close by noting that there may also be very special behavior of the $w$-sequence modulo 8. (Indeed, the data strongly suggest this.) Obviously, this would then imply congruences modulo 16 for the numbers $u(n)$.

Acknowledgments. The first two authors would like to thank the Danish Research Council (FNU) for the support of their collaboration. Thanks go also to a referee for remarks on alternative proofs. 


\section{REFERENCES}

[1] R. F. Churchrouse, Congruence properties of the binary partition function, Proc. Camb. Phil. Soc. 66 (1969), 371-376

[2] G. James, A. Kerber, The representation theory of the symmetric group. Encyclopedia of Mathematics and its Applications, 16, Addison-Wesley, Reading, Mass., 1981

[3] J.B. Olsson, Sign conjugacy classes in symmetric groups, J. Algebra 322 (2009), 2793-2800

[4] Ø. Rødseth, J. A. Sellers, Binary partitions revisited, J. Comb. Thy. Ser. A 98 (2002), $33-45$

[5] N.J.A. Sloane, J. A. Sellers, On non-squashing partitions, Discr. Math. 294 (2005), 259274.

[6] R. P. Stanley, Enumerative Combinatorics, vol. 2, Cambridge University Press, New York/Cambridge, 1999

[7] The On-Line Encyclopedia of Integer Sequences, published electronically at http://oeis.org. 2011

Institut für Algebra, Zahlentheorie und Diskrete Mathematik, Leibniz Universität Hannover, Welfengarten 1, D-30167 Hannover, Germany

E-mail address: bessen@math.uni-hannover.de

Department of Mathematical Sciences, University of Copenhagen, Universitetsparken 5,DK-2100 Copenhagen $\varnothing$, Denmark

E-mail address: olsson@math.ku.dk

Department of Mathematics, Penn State University, 104 McAllister Building, UniVERSITY PARK, PA 16802, USA

E-mail address: sellersj@math.psu.edu 